\title{
An Approximate Analytical Method of the Nonlinear Vibroacoustic Coupling System
}

\author{
Qizheng Zhou, Deshi Wang, and Yongyong Zhu \\ Department of Weaponry Engineering, Naval University of Engineering, Wuhan 430033, China \\ Correspondence should be addressed to Qizheng Zhou; zqizheng@sina.cn
}

Received 24 September 2013; Revised 3 April 2014; Accepted 5 May 2014; Published 22 May 2014

Academic Editor: Peijun Xu

Copyright (C) 2014 Qizheng Zhou et al. This is an open access article distributed under the Creative Commons Attribution License, which permits unrestricted use, distribution, and reproduction in any medium, provided the original work is properly cited.

\begin{abstract}
An approximate analytical method of the nonlinear vibroacoustic coupling system is proposed for the first time. Taking the Duffing oscillator-plate-medium system as an example, the nonlinear vibroacoustic coupling equations are developed using variational principle. The two major difficulties which lie in solving the coupling equations are the uncertain motion of the oscillator and the surface acoustic pressure on the plate, a system for which the fluid-structure coupling cannot be neglected. Based on the incremental harmonic balance (IHB) method, the motion of the oscillator is expressed in the form of the Fourier series, and then the modal expression method and the incoherent assumption are employed to discretize the displacement and the surface pressure of the plate. Then the approximate analytical solution is given by the IHB method. The characteristics of acoustic radiation and surface quadratic velocity of the plate, the nonlinear characteristics of oscillator, and the influence of the excitation frequency and the nonlinear stiffness on the results are investigated by the numerical simulation. The results show that the excitation at the frequency close to the natural frequency of the oscillator can produce a significant response of the third-harmonic generation which determines the vibroacoustic characteristics of the plate.
\end{abstract}

\section{Introduction}

The analytical solution of the nonlinear vibroacoustics coupling system is significant to the control of the vibration and acoustic radiation of structures under nonlinear excitations. The vibration isolation system as the typical supporting structure frequently encountered in many industrial applications transmits the excitation force to the radiator, and then the force excites the vibration and acoustic radiation of the radiator. The nonlinear vibration isolation mechanism is already clear, while the research on the acoustic radiation mechanism of the structure under the nonlinear vibration isolation is few. For the nonlinear vibroacoustic coupling system consisting of the nonlinear vibration isolation, plate, and acoustic medium, it is hard to obtain the exact analytical solutions due to the nonlinear factors, the vibration coupling of the oscillator and the plate, and the acoustic coupling of the plate and the acoustic medium. Although it could be solved by the numerical algorithms such as the finite element method (FEM) and the boundary element method (BEM), the results cannot represent the general rule. Therefore, this paper will propose an approximate analytical approach to the nonlinear vibroacoustic coupling system.

In the nonlinear vibroacoustic coupling system, the nonlinear oscillator's vibration is the excitation source of the radiator, the supporting structure is the sound channel to transmit the vibration, the surface wave of the radiator disturbs the surrounding acoustic medium, and then the acoustic pressure comes into being. On the other hand, the vibroacoustic coupling of the acoustic pressure and the plate's vibration affects the vibration of the oscillator. Hence, the research of the nonlinear vibroacoustic coupling system is an interdisciplinary issue between the branch of the mechanics and the acoustics. In the field of vibration and acoustic radiation, the dynamic response of the structure could be determined by the classical structure mechanics, and then the acoustic radiation characteristics could be obtained by the basis of compatibility condition between the structure surface and the acoustic medium. For a plate set in an infinite baffle, the radiated acoustic field can be calculated by a Rayleigh integral approach [1]. There are two common approaches to determine the radiation resistance, or efficiency. The first is 
to integrate the far-field acoustic intensity over a hemisphere enclosing the plate. The other is to integrate the acoustic intensity over the surface of vibrating plate. For a detailed study of the acoustic radiation it is necessary to derive expressions for the radiation resistance of the particular structural mode shapes of the plate. Maidanik [2] first proposed several approximate formulae for calculating the radiation resistance of vibrating plates and plate-like structures set in an infinite baffle. Whenever a closed form expression for the power radiated cannot be found for a particular source of interest, Williams [3] developed a power series expansion of the acoustic power radiating from baffled or unbaffled planar sources in terms of the various moments of the given velocity distribution in the source plane. The series expansion in terms of the Fourier transformed velocity and its derivatives in wave number space was used to derive approximate expressions for the power radiating at low frequencies from a mode of a rectangular plate with three different types of boundary conditions. In order to solve the singular integration in calculating the radiation resistance of a simple supported rectangular plate with an arbitrary acoustic wave number, $\mathrm{Li}$ and Gibeling [4] and $\mathrm{Li}$ [5] gave an asymptotic solution based on [3], in the form of a power series of the nondimensional acoustic wave number, to calculate the self- and mutual radiation resistance in the entire frequency range, which is a simple and general way and is theoretically good for any acoustic wave numbers or frequencies. On the research of the structural nonlinear vibration, it deals with the geometrically nonlinear problem using the strain-stress relationship. The methods mainly are the analytic method (for the elemental basic structure in [6]), the FEM in [7], and the BEM in [8]), while there are few works on the acoustic radiation of the nonlinear structure.

In the vibroacoustic coupling theory, the former work mainly considered the basic structures, and the latter was the stiffened structures. For these structures, the dynamical equations of the vibroacoustic coupling system were established based on the variational principle; then the modal expression method, the Fourier transform method, and the stationary phase method were used to solve the coupling equations. Crighton and Maidanik [9] presented an analytical study of the interaction between incident wave fields and a single rib on a fluid-loaded panel, and then Crighton illustrated that the fluid loading has many varied and profound influence on the vibration of structures and on their acoustic fields in analytical and physical terms through simple models in [10]. Burroughs and Hallander [11] derived the analytical expressions for the far-field acoustic radiation from a fluid-loaded circular cylindrical shell reinforced with two sets of parallel periodic ribs and excited by seven types of mechanical point drives. Recently, a semianalytical model employs the modal expansion technique in order to investigate the vibroacoustic behavior of stiffened composite panels under airborne and structure borne excitations in [12]. And Chen et al. [13] implemented a complicated multiple compartment shell structure in water and concluded that the sound pressure caused by multiple excitations can be approximated as the incoherent superposition of those caused by each excitation separately.
The main methods to investigate the nonlinear vibration system are the theoretical analysis method and the experimental method in [14]. The theoretical method is the basic method including the qualitative theory and the quantitative method. The perturbation techniques including the Lindstedt-Poincare method, the method of multiple scales, and the KBM method are a classical quantitative method to investigate the weakly nonlinear system. Small parameters are essentially assumed so that perturbations can be carried out. The IHB method can give accurate results without difficulty in increasing the accuracy of the results and without considering the parameter range. In the IHB method, nonlinear differential equations are reduced to a set of linearized incremental algebraic equations in terms of the Fourier coefficients. Lau [15] originally presented the analyses of periodic structural vibrations by the method and it has also been successfully applied to various types of nonlinear dynamics. For example, the generalized incremental Hamilton's principle and element formulation are used to study the nonlinear vibration of thin elastic plates [16]. The IHB method is employed to investigate the Hopf bifurcation and limit cycle [17], and the IHB method is also used with the fast Fourier algorithm to research the steady-state oscillation of hysteretic differential model [18]. Recently, Sze et al. [19] used the IHB method to study the nonlinear vibration of axially moving beams, and Hall et al. [20] used the IHB method to investigate the computational fluid dynamics problems. By considering typical examples they have shown that the method is very effective for analyzing structural and mechanical systems with nonlinear characteristics.

Given the IHB method expanding the periodic solution into the Fourier series and the relationship between the surface acoustic pressure and the excitation frequency, the IHB method and the modal expression method are employed to derive the approximate analytical solution of the vibroacoustic coupling system. The Duffing oscillator-platemedium system as an example and the vibroacoustic coupling equations will be obtained by the variational principle; then the displacement and the surface pressure of the plate could be expressed in the superposition form of the normal modes and the temporal pulse shapes based on the motion form of the oscillator, the modal expression method, and the incoherent assumption. Then the IHB method is used to derive the approximate analytical solution of the nonlinear multi-degree-of-freedom system.

\section{Physical Model and Nonlinear Vibroacoustic Coupling Equation}

The example considered is a simply supported rectangular plate under a nonlinear oscillator's excitation in an infinite rigid baffle, as shown in Figure 1. Properties of the plate include its length $a$, width $b$, thickness $h$, density $\rho$, Young's modulus $E$, and Poisson ratio $\mu$. The mass of the equipment is $m$, and the excitation force is $f(t)=F \cos (\omega t)$. The linear and nonlinear stiffness coefficients of supporting spring are $k_{1}$ and $k_{3}$, respectively, and the location of spring on the thin plate is $\left(x_{0}, y_{0}, 0\right)$. The displacement of the oscillator is 


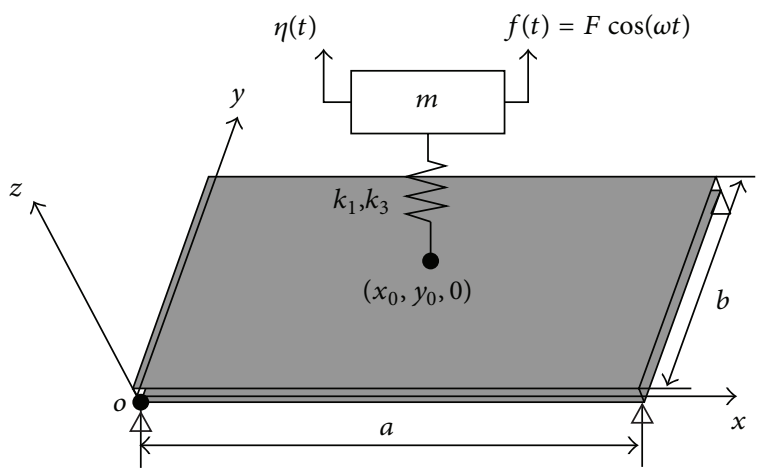

Figure 1: Vibroacoustic model of the plate under a nonlinear oscillator's excitations.

denoted by $\eta(t)$. The vibration equation of equipment is the typical Duffing equation when neglecting the effect of plate's vibration. Assuming the motion of the plate just accounting for the transverse displacement $w$, the nonlinear vibroacoustic coupling equation of the plate under the oscillator's excitation will be derived according to variational principle.

Based on thin plate theory [21], the strain energy of the plate is

$$
\begin{aligned}
V_{p}=\frac{1}{2} \int_{0}^{a} \int_{0}^{b}( & D\left(\nabla^{2} w\right)^{2}-2(1-\mu) \\
& \left.\times\left(\frac{\partial^{2} w}{\partial x^{2}} \frac{\partial^{2} w}{\partial y^{2}}-\left(\frac{\partial^{2} w}{\partial x \partial y}\right)^{2}\right)\right) d x d y,
\end{aligned}
$$

where $D=E h^{3} / 12\left(1-\mu^{2}\right)$ is the flexural stiffness and $\nabla^{2}=$ $\partial^{2} / \partial x^{2}+\partial^{2} / \partial y^{2}$ is the Laplace operators. The kinetic energy of the plate is

$$
T_{p}=\frac{1}{2} \int_{0}^{a} \int_{0}^{b} \rho h \dot{w}^{2} d x d y
$$

The elastic potential energy of the spring is

$$
V_{S}=\frac{1}{2} k_{1}\left(\eta-w\left(x_{0}, y_{0}, t\right)\right)^{2}+\frac{1}{4} k_{3}\left(\eta-w\left(x_{0}, y_{0}, t\right)\right)^{4} .
$$

The kinetic energy of the equipment is

$$
T_{o}=\frac{1}{2} m \dot{\eta}(t)^{2} .
$$

The work done by the external forces includes the excitation forces and the acoustic pressure exerted by the fluid on the plate. The acoustic pressure on the plate is $p(x, y, 0, t)$, and then the work due to the fluid loading is

$$
V_{f}=\int_{0}^{a} \int_{0}^{b} p(x, y, 0, t) w(x, y, t) d x d y .
$$

The work done by the excitation force on the equipment is

$$
W_{f}=f(t) \eta(t) .
$$

Using variational principle, the nonlinear vibroacoustic coupling equation of the plate-oscillator system is

$$
\delta \int_{t_{1}}^{t_{2}}\left(T_{p}+T_{o}-V_{p}-V_{S}\right) d t+\int_{t_{1}}^{t_{2}} \delta\left(V_{f}+W_{f}\right) d t=0 .
$$

Then,

$$
\begin{aligned}
& m \ddot{\eta}(t)+k_{1}\left(\eta(t)-w\left(x_{0}, y_{0}, t\right)\right) \\
& \quad+k_{3}\left(\eta(t)-w\left(x_{0}, y_{0}, t\right)\right)^{3}=F \cos (\omega t), \\
& D\left(\left(\nabla^{2} w\right)^{2}-2(1-\mu)\left(\frac{\partial^{2} w}{\partial x^{2}} \frac{\partial^{2} w}{\partial y^{2}}-\left(\frac{\partial^{2} w}{\partial x \partial y}\right)^{2}\right)\right) \\
& \quad+\rho h w^{2} \frac{\partial^{2} w}{\partial t^{2}}+p(x, y, 0, t) \\
& \quad-\left(k_{1}\left(\eta(t)-w\left(x_{0}, y_{0}, t\right)\right)+k_{3}\left(\eta(t)-w\left(x_{0}, y_{0}, t\right)\right)^{3}\right) \\
& \quad \times \delta\left(x-x_{0}\right) \delta\left(y-y_{0}\right)=0 .
\end{aligned}
$$

Equations (8a) and (8b) show that the nonlinear vibroacoustic coupling equation is the combination of the discrete system and the continuous system. Due to the thrice nonlinear stiffness, there are some strong nonlinear terms in the coupling equation. Therefore, the IHB method and the modal expansion method are employed to derive the approximately analytical solution of the coupling equation.

\section{Solution}

In this section, the IHB method and the modal expression method are formulated to derive the approximate analytical solution of the nonlinear vibroacoustic coupling system. The new dimensionless time variable $\tau$ is defined as

$$
\tau=\omega t .
$$

Substituting (9) into (8a) and (8b), then

$$
\begin{aligned}
& m \omega^{2} \eta^{\prime \prime}(\tau)+k_{1}\left(\eta(\tau)-w\left(x_{0}, y_{0}, \tau\right)\right)+k_{3} \\
& \quad \times\left(\eta(\tau)-w\left(x_{0}, y_{0}, \tau\right)\right)^{3}=F \cos (\tau), \\
& D\left(\left(\nabla^{2} w\right)^{2}-2(1-\mu)\left(\frac{\partial^{2} w}{\partial x^{2}} \frac{\partial^{2} w}{\partial y^{2}}-\left(\frac{\partial^{2} w}{\partial x \partial y}\right)^{2}\right)\right) \\
& \quad+\rho h \omega^{2} \frac{\partial^{2} w}{\partial \tau^{2}}+p(x, y, 0, \tau) \\
& \quad-\left(k_{1}\left(\eta(\tau)-w\left(x_{0}, y_{0}, \tau\right)\right)+k_{3}\left(\eta(\tau)-w\left(x_{0}, y_{0}, \tau\right)\right)^{3}\right) \\
& \quad \times \delta\left(x-x_{0}\right) \delta\left(y-y_{0}\right)=0 .
\end{aligned}
$$

There are only odd harmonic terms of cosine in the solution of Duffing equation in [22], so $\eta(\tau)$ can be expressed as

$$
\eta(\tau)=a_{1} \cos (\tau)+a_{3} \cos (3 \tau)+\cdots .
$$


Based on the modal expansion method, the transverse vibration displacement $w$ can be expressed in terms of admissible trigonometric trial functions [21]:

$$
\begin{gathered}
w(x, y, \tau)=\sum_{m=0}^{\infty} \sum_{n=0}^{\infty} w_{m n} \\
=\sum_{m=0}^{\infty} \sum_{n=0}^{\infty}\left(a_{m n 1} \cos (\tau)+a_{m n 3} \cos (3 \tau)+\cdots\right) \\
\quad \times \sin k_{m} x \sin k_{n} y
\end{gathered}
$$

where $k_{m}=m \pi / a$ and $k_{n}=n \pi / b$ are determined by the simply supported conditions.

Based on Rayleigh integral formulation, the acoustic pressure of the observation point above the plate is

$$
\begin{aligned}
& p(x, y, z, \tau) \\
& =-\sum_{m=1}^{\infty} \sum_{n=1}^{\infty} \frac{\omega^{2} \rho_{0}}{2 \pi} \\
& \quad \times \iint_{S} \frac{\sin k_{m} x^{\prime} \sin k_{n} y^{\prime}}{\left|r-r^{\prime}\right|} \\
& \quad \times\left(a_{m n 1} \cos (\tau) e^{-i k\left|r-r^{\prime}\right|}\right. \\
& \left.\quad+9 a_{m n 3} \cos (3 \tau) e^{-i 3 k\left|r-r^{\prime}\right|}+\cdots\right) d x^{\prime} d y^{\prime},
\end{aligned}
$$

where $\left|r-r^{\prime}\right|=\sqrt{\left(x-x^{\prime}\right)^{2}+\left(y-y^{\prime}\right)^{2}+\left(z-z^{\prime}\right)^{2}}$ is the distance of observation point and $k, \omega$, and $\rho_{0}$ are the wave number, the excitation frequency, and the medium density.

Inserting (11)-(13) into (10a) and (10b), multiplying the second equation by $\sin k_{m} x \sin k_{n} y$, integrating over the plate surface, and using the orthogonality of the plate modes, (10a) and (10b) can be transformed as

$$
\begin{aligned}
& -m \omega^{2}\left(a_{1} \cos (\tau)+9 a_{3} \cos (3 \tau)+\cdots\right)+k_{1} \\
& \times\left(\left(a_{1}-\sum_{m=1}^{\infty} \sum_{n=1}^{\infty} c_{m n} a_{1 m n}\right) \cos (\tau)\right. \\
& \left.+\left(a_{3}-\sum_{m=1}^{\infty} \sum_{n=1}^{\infty} c_{m n} a_{3 m n}\right) \cos (3 \tau)+\cdots\right)+k_{3} \\
& \times\left(\left(a_{1}-\sum_{m=1}^{\infty} \sum_{n=1}^{\infty} c_{m n} a_{1 m n}\right) \cos (\tau)\right. \\
& \left.+\left(a_{3}-\sum_{m=1}^{\infty} \sum_{n=1}^{\infty} c_{m n} a_{3 m n}\right) \cos (3 \tau)+\cdots\right)^{3}
\end{aligned}
$$

$=F \cos (\tau)$,

$$
\begin{aligned}
& b_{m n}\left(a_{1 m n} \cos (\tau)+a_{3 m n} \cos (3 \tau)+\cdots\right) \\
& -\bar{m} \omega^{2}\left(a_{1 m n} \cos (\tau)+9 a_{3 m n} \cos (3 \tau)+\cdots\right)-\frac{4 \omega}{a b D} \\
& \times\left(Z_{1 m n} a_{1 m n} \cos (\tau)+9 Z_{3 m n} a_{3 m n} \cos (3 \tau)+\cdots\right)-\bar{c}_{m n} \\
& \times\left\{k _ { 1 } \left(\left(a_{1}-\sum_{m=1}^{\infty} \sum_{n=1}^{\infty} c_{m n} a_{1 m n}\right) \cos (\tau)\right.\right. \\
& \left.+\left(a_{3}-\sum_{m=1}^{\infty} \sum_{n=1}^{\infty} c_{m n} a_{3 m n}\right) \cos (3 \tau)+\cdots\right) \\
& +k_{3}\left(\left(a_{1}-\sum_{m=1}^{\infty} \sum_{n=1}^{\infty} c_{m n} a_{1 m n}\right) \cos (\tau)\right. \\
& \left.\left.+\left(a_{3}-\sum_{m=1}^{\infty} \sum_{n=1}^{\infty} c_{m n} a_{3 m n}\right) \cos (3 \tau)+\cdots\right)^{3}\right\}
\end{aligned}
$$

$=0$,

where $\bar{m}=\rho h / D, b_{m n}=\left(k_{m}^{2}+k_{n}^{2}\right)^{2}, c_{m n}=\sin k_{m} x_{0} \sin k_{n} y_{0}$, $\bar{c}_{m n}=4 c_{m n} / a b D$, and $Z_{1 m n}$ and $Z_{3 m n}$ are the self-radiation resistances of the plate (see Appendix A). The mutual radiation resistances are neglected due to the little contributions to the acoustic pressure.

When the effect of the plate's vibration is neglected, (14) becomes

$$
\begin{aligned}
& -m \omega^{2}\left(a_{1} \cos (\tau)+9 a_{3} \cos (3 \tau)+\cdots\right) \\
& \left.+k_{1}\left(a_{1} \cos (\tau)\right)+a_{3} \cos (3 \tau)+\cdots\right) \\
& +k_{3}\left(a_{1} \cos (\tau)+a_{3} \cos (3 \tau)+\cdots\right)^{3}=F \cos (\tau) .
\end{aligned}
$$

The IHB method used to solve (15) is detailed in [20].

The second step in applying the IHB method to obtain the periodic solutions is to add increments from some initial guess of the solution of (14) $\eta_{0}, \omega_{0}, w_{0}$ to the actual solution $\eta^{*}, \omega^{*}, w^{*}$, iteratively. Then $\eta, w$ are expanded in a finite Fourier series in the form

$$
\begin{gathered}
\omega=\omega_{0}+\Delta \omega \\
\eta(\tau)=\left(a_{10}+\Delta a_{1}\right) \cos (\tau)+\left(a_{30}+\Delta a_{3}\right) \cos (3 \tau)+\cdots, \\
w(x, y, \tau) \\
=\sum_{m=1}^{\infty} \sum_{n=1}^{\infty}\left(\left(a_{1 m n 0}+\Delta a_{1 m n}\right) \cos (\tau)\right. \\
\left.\quad+\left(a_{3 m n 0}+\Delta a_{3 m n}\right) \cos (3 \tau)+\cdots\right) \\
\quad \times \sin k_{m} x \sin k_{n} y .
\end{gathered}
$$


For simplification, two Fourier series in the form are used. Substituting (16) into (14) and expanding the resulting product of Fourier series into a single summation of cosines, then using Galerkin procedure, (14) becomes

$$
\begin{aligned}
& -m \omega_{0}^{2} \Delta a_{1}+\left(k_{1}+k_{3}\left(\frac{9}{4} \bar{a}_{1}^{2}+\frac{3}{2} \bar{a}_{3}^{2}+\frac{3}{2} \bar{a}_{1} \bar{a}_{3}\right)\right) \\
& \times \Delta \bar{a}_{1}+k_{3}\left(\frac{3}{4} \bar{a}_{1}^{2}+3 \bar{a}_{1} \bar{a}_{3}\right) \Delta \bar{a}_{3} \\
& =R_{1}+2 m \omega_{0} a_{1} \Delta \omega \text {, } \\
& -9 m \omega_{0}^{2} \Delta a_{3}+k_{3}\left(\frac{3}{4} \bar{a}_{1}^{2}+3 \bar{a}_{1} \bar{a}_{3}\right) \Delta \bar{a}_{1} \\
& +\left(k_{1}+k_{3}\left(\frac{3}{2} \bar{a}_{1}^{2}+\frac{9}{4} \bar{a}_{3}^{2}\right)\right) \Delta \bar{a}_{3} \\
& =R_{3}+18 m \omega_{0} a_{3} \Delta \omega, \\
& \left(b_{m n}-\bar{m} \omega \omega_{0}^{2}-\frac{4 \omega_{0} Z_{1 m n}}{a b D}\right) \Delta a_{1 m n} \\
& -\bar{c}_{m n}\left\{\left(k_{1}+k_{3}\left(\frac{9}{4} \bar{a}_{1}^{2}+\frac{3}{2} \bar{a}_{3}^{2}+\frac{3}{2} \bar{a}_{1} \bar{a}_{3}\right)\right)\right. \\
& \left.\times \Delta \bar{a}_{1}+k_{3}\left(\frac{3}{4} \bar{a}_{1}^{2}+3 \bar{a}_{1} \bar{a}_{3}\right) \Delta \bar{a}_{3}\right\} \\
& =R_{1 m n}+\left(2 \bar{m} \omega_{0}-\frac{4 Z_{1 m n}}{a b D}\right) a_{1 m n} \Delta \omega, \\
& \left(b_{m n}-9\left(\bar{m} \omega_{0}^{2}+\frac{4 \omega_{0} Z_{3 m n}}{a b D}\right)\right) \Delta a_{3 m n} \\
& -\bar{c}_{m n}\left\{k_{3}\left(\frac{3}{4} \bar{a}_{1}^{2}+3 \bar{a}_{1} \bar{a}_{3} \Delta \bar{a}_{1}\right)\right. \\
& \left.+\left(k_{1}+k_{3}\left(\frac{3}{2} \bar{a}_{1}^{2}+\frac{9}{4} \bar{a}_{3}^{2}\right)\right) \Delta \bar{a}_{3}\right\} \\
& =R_{3 m n}+9\left(2 \bar{m} \omega_{0}-\frac{4 Z_{3 m n}}{a b D}\right) a_{3 m n} \Delta \omega, \\
& R_{1}=F+m \omega \omega_{0}^{2} a_{1}-k_{1} \bar{a}_{1}-k_{3}\left(\frac{3}{4} \bar{a}_{1}^{3}+\frac{3}{4} \bar{a}_{1}^{2} \bar{a}_{3}+\frac{3}{2} \bar{a}_{1} \bar{a}_{3}^{2}\right), \\
& R_{3}=9 m \omega_{0}^{2} a_{3}-k_{1} \bar{a}_{3}-k_{3}\left(\frac{1}{4} \bar{a}_{1}^{3}+\frac{3}{4} \bar{a}_{3}^{3}+\frac{3}{2} \bar{a}_{1}^{2} \bar{a}_{3}\right), \\
& R_{1 m n}=\left(\bar{m} \omega_{0}^{2}+\frac{4 \omega_{0} Z_{1 m n}}{a b D}-b_{m n}\right) a_{1 m n}+\bar{c}_{m n} \\
& \times\left(k_{1} \bar{a}_{1}+k_{3}\left(\frac{3}{4} \bar{a}_{1}^{3}+\left(\frac{3}{4} \bar{a}_{1}^{2} \bar{a}_{3}+\frac{3}{2} \bar{a}_{1} \bar{a}_{3}^{2}\right)\right)\right), \\
& R_{3 m n}=\left(9\left(\bar{m} \omega_{0}^{2}+\frac{4 \omega_{0} Z_{3 m n}}{a b D}\right)-b_{m n}\right) a_{3 m n}+\bar{c}_{m n} \\
& \times\left(k_{1} \bar{a}_{3}+k_{3}\left(\frac{1}{4} \bar{a}_{1}^{3}+\frac{3}{4} \bar{a}_{3}^{3}+\frac{3}{2} \bar{a}_{1}^{2} \bar{a}_{3}\right)\right),
\end{aligned}
$$

where $\bar{a}_{1}=a_{10}-\sum_{m=1}^{\infty} \sum_{n=1}^{\infty} c_{m n} a_{1 m n 0}, \bar{a}_{3}=a_{30}-$ $\sum_{m=1}^{\infty} \sum_{n=1}^{\infty} c_{m n} a_{3 m n 0}, \Delta \bar{a}_{1}=\Delta a_{1}-\sum_{m=1}^{\infty} \sum_{n=1}^{\infty} c_{m n} \Delta a_{1 m n}$,
$\Delta \bar{a}_{3}=\Delta a_{3}-\sum_{m=1}^{\infty} \sum_{n=1}^{\infty} c_{m n} \Delta a_{3 m n} \cdot R_{1}, R_{3}, R_{1 m n}$, and $R_{3 m n}$ are called the residual terms. If the solution $\eta_{0}, \omega_{0}, w_{0}$ is an exact solution of (17a), (17b), (17c), and (17d), then the residual terms are zero. In this sense, the residual term can be used to control the accuracy in the computation. It is noted that (17a), (17b), (17c), and (17d) represent a set of $2(M \times$ $N+1)$ linear algebraic equations $(M, N$ are the truncation numbers). Rewrite (17a), (17b), (17c), and (17d) in matrix form

$$
\mathbf{K} \Delta \mathbf{a}=\mathbf{R},
$$

where $\Delta \mathbf{a}=\left[\Delta a_{1}, \Delta a_{3}, \Delta a_{111}, \Delta a_{112}, \ldots, \Delta a_{1 M N}, \Delta a_{311}, \Delta a_{312}\right.$, $\left.\ldots, \Delta a_{3 M N}\right]^{T}$ and the matrix $\mathbf{K}$ and the vector $\mathbf{R}$ are shown in Appendix B.

It is noted that the number of unknowns is $2(M \times N+$ 1) +1 while the number of algebraic equations is $2(M \times N+$ $1)$. The solution process begins with a guessed solution. The solution of (19) is then solved point-by-point by incrementing the frequency $\omega$. The Newton-Raphson iterative method can be employed. By solving the nonlinear vibroacoustic coupling equation, one can obtain the mode amplitude of $w$. Hence, one can further obtain the surface quadratic velocity on the surface of plate.

The following quantities will be used to describe the vibroacoustic characteristics of the plate. The surface quadratic velocity is a space-time average of the square vibrational velocity of plate; it is defined by

$$
\begin{aligned}
V^{2} & =\frac{1}{S} \frac{1}{T} \int_{0}^{T} \iint_{S}\left|\frac{\partial w(x, y, t)}{\partial t}\right|^{2} d S d t \\
& =\frac{\omega^{2}}{8} \sum_{m=1}^{\infty} \sum_{n=1}^{\infty}\left(\left|a_{1 m n}\right|^{2}+9\left|a_{3 m n}\right|^{2}\right),
\end{aligned}
$$

where $T=2 \pi / \omega$ and $S$ is the surface-area of plate.

The radiated acoustic power, which is the integration of the active intensity over the surface of plate, is defined by

$$
\begin{gathered}
W=\frac{1}{T} \int_{0}^{T} \iint_{S} P(x, y, 0, t) \frac{\partial w(x, y, t)}{\partial t} d S d t \\
=\frac{\omega^{2}}{2} \sum_{m=1}^{\infty} \sum_{n=1}^{\infty}\left(a_{1 m n} \operatorname{Re}\left[Z_{1 m n}\right] a_{1 m n}^{*}\right. \\
\left.+9 a_{3 m n} \operatorname{Re}\left[Z_{3 m n}\right] a_{3 m n}^{*}\right),
\end{gathered}
$$

where Re denotes the real part and $*$ is the complex conjugate. The radiated acoustic power levels and the surface quadratic velocity levels are defined as

$$
L_{W}(\omega)=10 \lg \frac{W}{W_{0}}, \quad L_{V}(\omega)=10 \lg \frac{\left\langle V^{2}\right\rangle}{v_{0}^{2}},
$$

where the reference acoustic power is $W_{0}=0.67 \times 10^{-18} \mathrm{~W}$ and the reference velocity is $v_{0}=5 \times 10^{-8} \mathrm{~m} / \mathrm{s}$.

\section{Numerical Simulation and Results Discussion}

In this section, the numerical simulation is used for investigating the nonlinear vibroacoustic coupling characteristics 


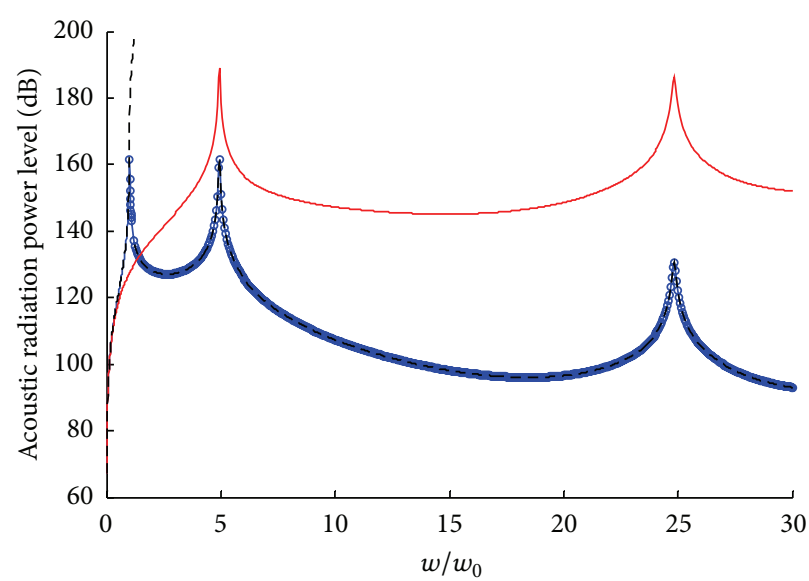

- Excitation force on the plate directly

$\rightarrow-\infty$ Neglecting nonlinear stiffness

- - - Considering the nonlinear stiffness

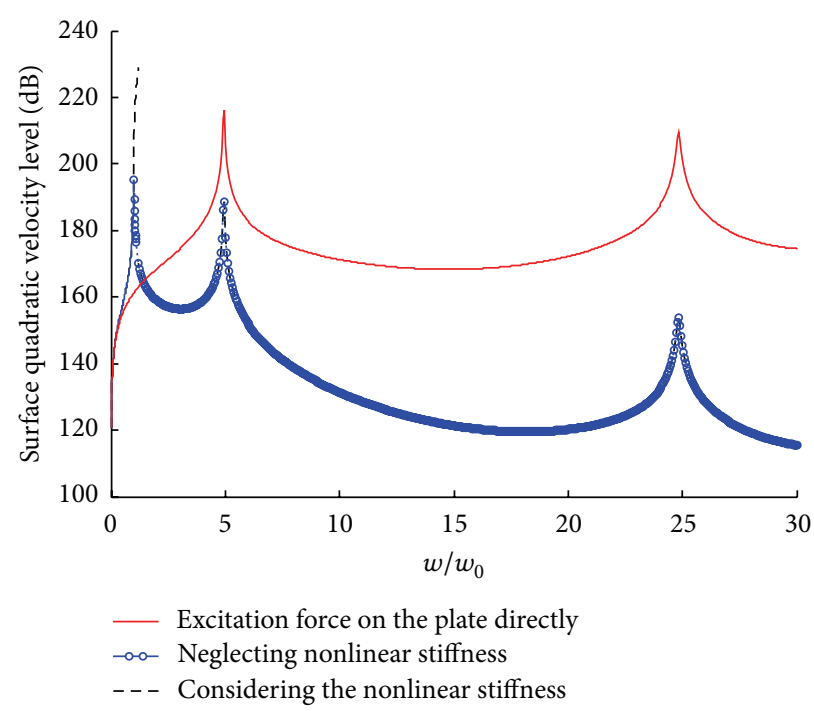

(b)

Figure 2: Influence of the supporting form on the vibration properties of the oscillator: (a) acoustic radiation power level and (b) surface quadratic velocity level.

of the system based on the proposed method. The simply supported rectangular plate under a nonlinear oscillator's excitation in an infinite rigid baffle is shown in Figure 1. The structural parameters of the plate in the numerical study are selected as $a=3 \mathrm{~m}, b=2 \mathrm{~m}$, and $h=0.002 \mathrm{~m}$ with a density of $7.8 \times 10^{3} \mathrm{~kg} / \mathrm{m}^{3}$, Young's modulus of $2.1 \times 10^{11} \mathrm{~N} / \mathrm{m}^{2}$, and Poisson's ratio of 0.3 . The plate is submerged in water with a density of $1000 \mathrm{~kg} / \mathrm{m}^{3}$ and the acoustic velocity of $1.5 \times$ $10^{3} \mathrm{~m} / \mathrm{s}$. The parameters of nonlinear oscillator are $m=10 \mathrm{~kg}$ and $k_{1}=1000 \mathrm{~N} / \mathrm{m}$, and the oscillator is fixed on the plate with the location of $(a / 3, b / 3,0)$. The amplitude of excitation force on the oscillator is $F=100 \mathrm{~N}$.

The vibroacoustic characteristics of the plate and the nonlinear vibration characteristics of the oscillator are shown in Figures 2 to 6 . The comparisons are concluded in the following subsections.

4.1. Vibroacoustic Properties of the Plate. The influence of the supporting form on the acoustic radiation power level and surface quadratic velocity level is illustrated in Figure 2. It is noted that the number of the peaks in the system with the supporting structure is one more than in the system without the supporting structure, and the peak value at the natural frequency of the oscillator is the largest one. The acoustic radiation power level and the surface quadratic velocity level of the plate under the excitation force which worked on the plate directly are lager $30-60 \mathrm{~dB}$ above $5 \omega_{0}\left(\omega_{0}=\sqrt{k_{1} / m}=\right.$ $10 \mathrm{rad} / \mathrm{s})$. Hence, putting the equipment on support structure is a good way to control the acoustic radiation and the vibration if the excitation frequency is above five times the oscillator's natural frequency.

Figure 3 presents the harmonic components on the vibroacoustic characteristics of the plate when $k_{3}=10 k_{1}$.
It can be seen that the acoustic radiation power level and the surface quadratic velocity level are dominated by the first harmonic component in most frequency bands. While the excitation at a frequency close to the natural frequency of the oscillator can produce a significant response at the third-harmonic generation, the vibroacoustic characteristics are determined by the third-harmonic generation. And it is clear that the number of peaks induced by third-harmonic component is more. The peak at $\omega_{0} / 3$ is induced by the third super harmonic resonance of the oscillator. The others are at the natural frequencies of plate, which is because the frequency of the third-harmonic component is three times the excitation frequency.

Figure 4 presents the influence of the nonlinear stiffness on the vibroacoustic characteristics of the plate. In the present example, the nonlinear stiffness has no key effect on the acoustic radiation power level and surface quadratic velocity level in most frequency bands. However at the oscillator's resonant region, as the nonlinear stiffness increases, the amplitudes increase.

4.2. Vibration Properties of the Oscillator. The effect of the plate's vibration on the nonlinear vibration properties of the oscillator is presented. Figure 5 compares the amplitudes of $a_{1}$ and $a_{3}$ calculated by the simple Duffing equation and the nonlinear vibroacoustic coupling equation (19). Figure 5 shows that $a_{1} \gg a_{3}$. It can be found that the vibration of the plate has no key influence on the vibration of Duffing oscillator, especially below and above the natural frequency $(10 \mathrm{rad} / \mathrm{s})$ of the oscillator. It is because that the amplitudes of the oscillator for these frequencies are so small that the nonlinear forces transmitted to the plate could not induce the plate's vibration effectively. Nevertheless at the 


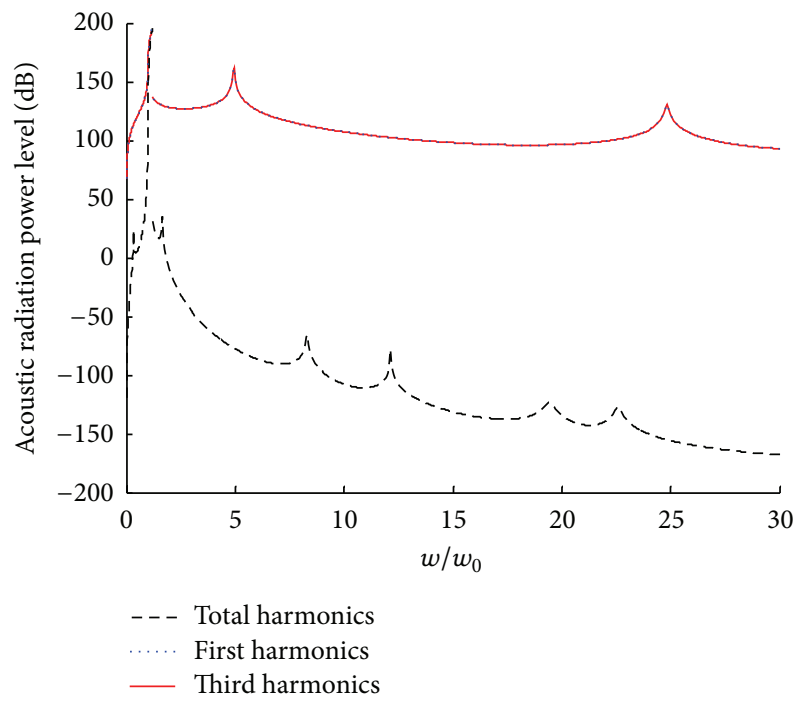

(a)

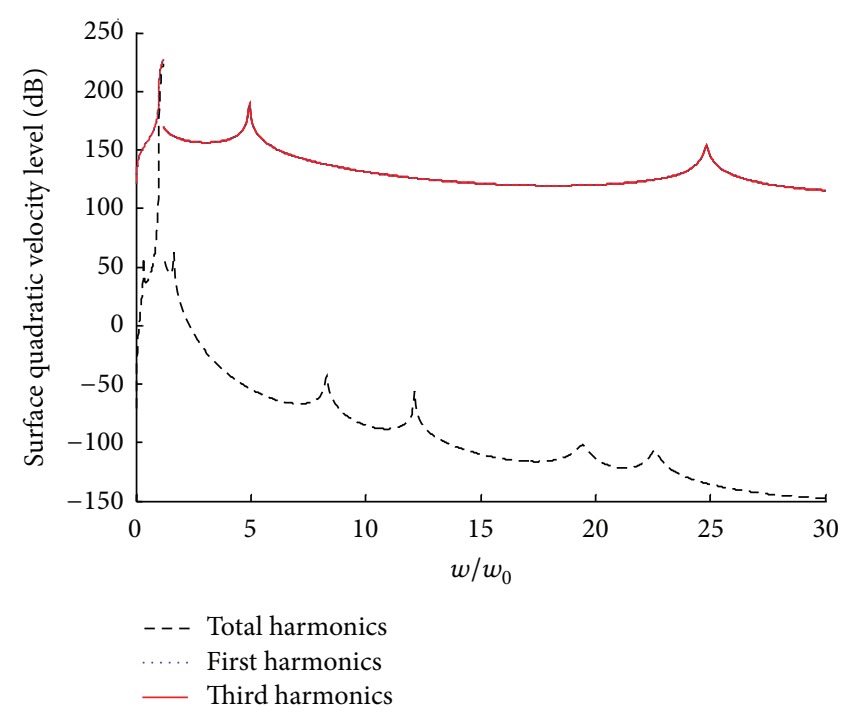

(b)

FiguRE 3: Influence of harmonic components on the vibroacoustic properties of the plate $\left(k_{3}=10 k_{1}\right)$ : (a) acoustic radiation power level and (b) surface quadratic velocity level.

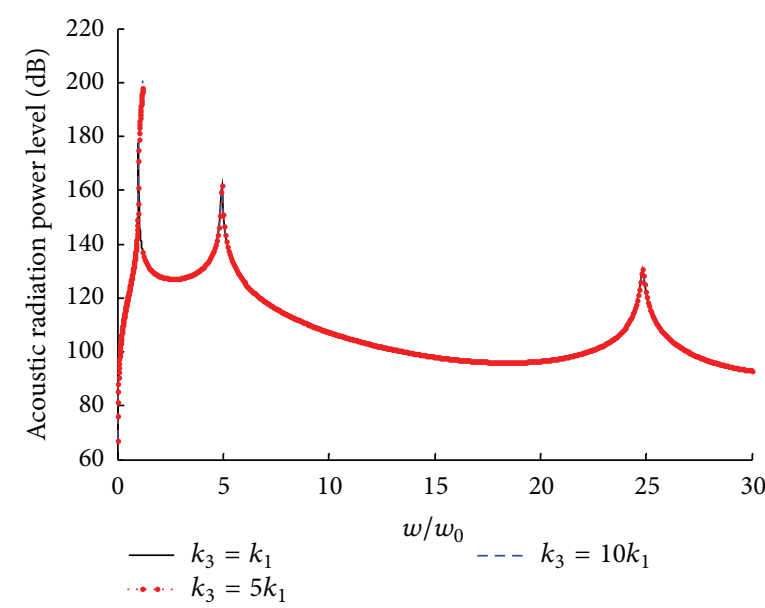

(a)

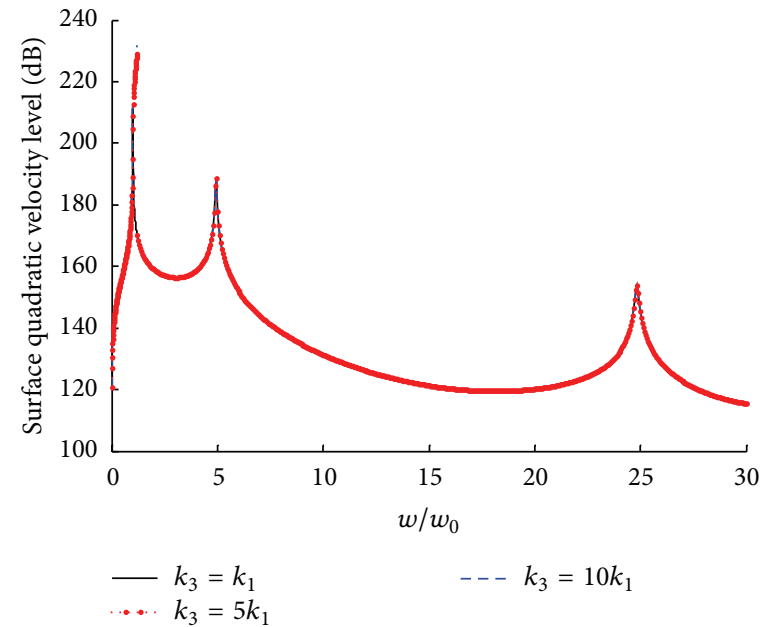

(b)

FIGURE 4: Influence of the nonlinear stiffness on the vibroacoustic properties of the plate: (a) acoustic radiation power level and (b) surface quadratic velocity level.

resonance region, the amplitudes are so large that the jump phenomenon of the Duffing oscillator is changed, and the resonance region of considering the plate's vibration is wider. This may be the main influence of the plate's vibration on the oscillator. It could be explained by the fact that the structural impedance of the system is increased by the plate, so the resonant prosperity of system is changed.

The oscillator's amplitudes of the plate-oscillator-medium system with different nonlinear stiffness are given in Figure 6.
Comparing the curves in Figure 6, it could be found that when the excitation frequency is above $20 \mathrm{rad} / \mathrm{s}$, the nonlinear stiffness has no evident influence on the amplitudes $a_{1}$ and $a_{3}$. Below $20 \mathrm{rad} / \mathrm{s}$, as the nonlinear stiffness increases, the unstable region and the amplitude $a_{3}$ rise, while the amplitude $a_{1}$ drops slightly.

Due to Figures 5 and 6 , it is worth noticing that, in the present example, the vibration of the plate could change the jump phenomenon of the Duffing oscillator, 


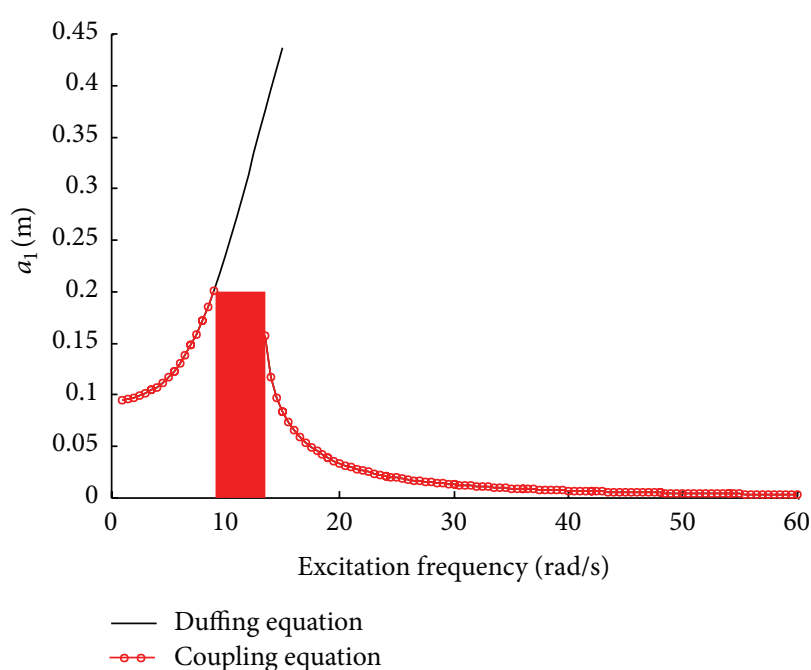

(a)

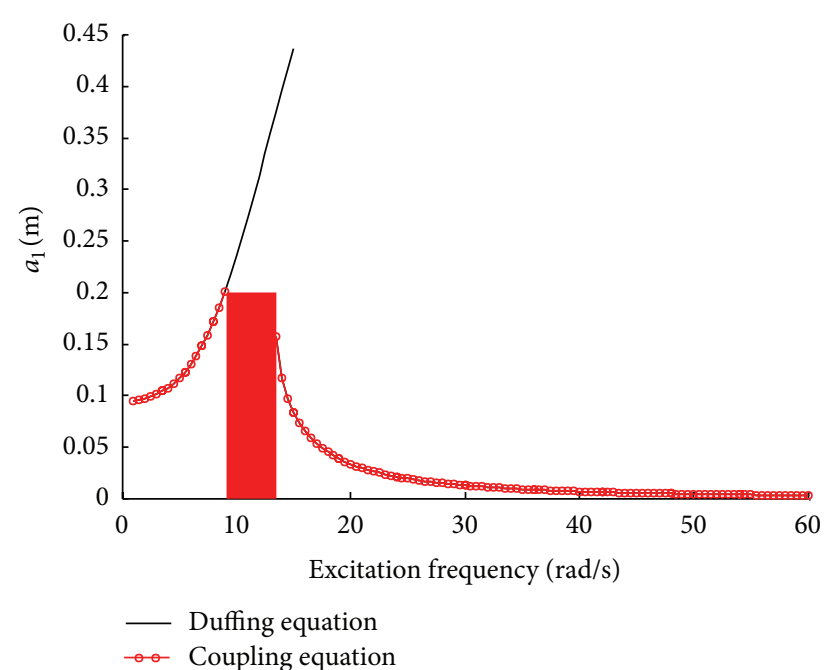

(b)

FIGURE 5: Influence of the plate's vibration on the vibration properties of the oscillator $\left(k_{3}=10 k_{1}\right)$; the shadow range is the unstable range: (a) the influence on $a_{1}$ and (b) the influence on $a_{3}$.

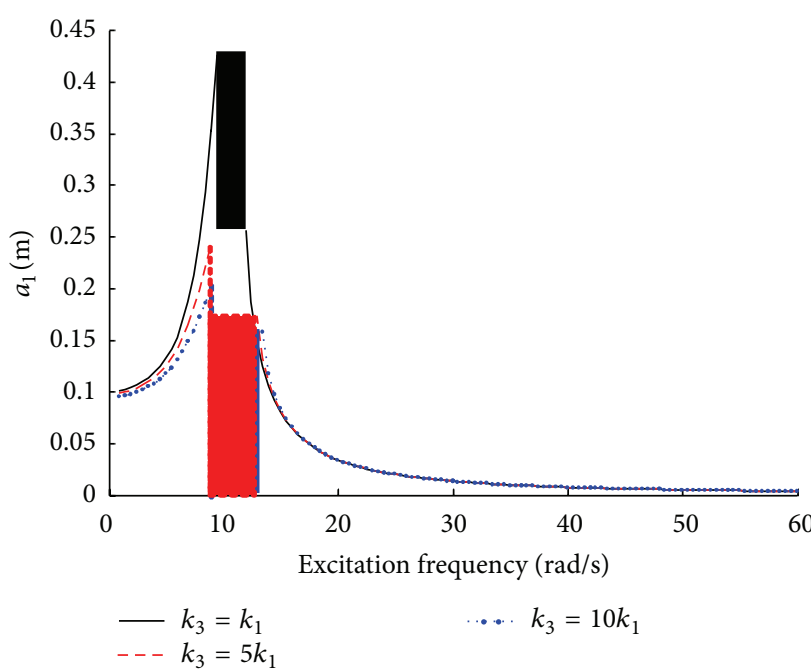

(a)

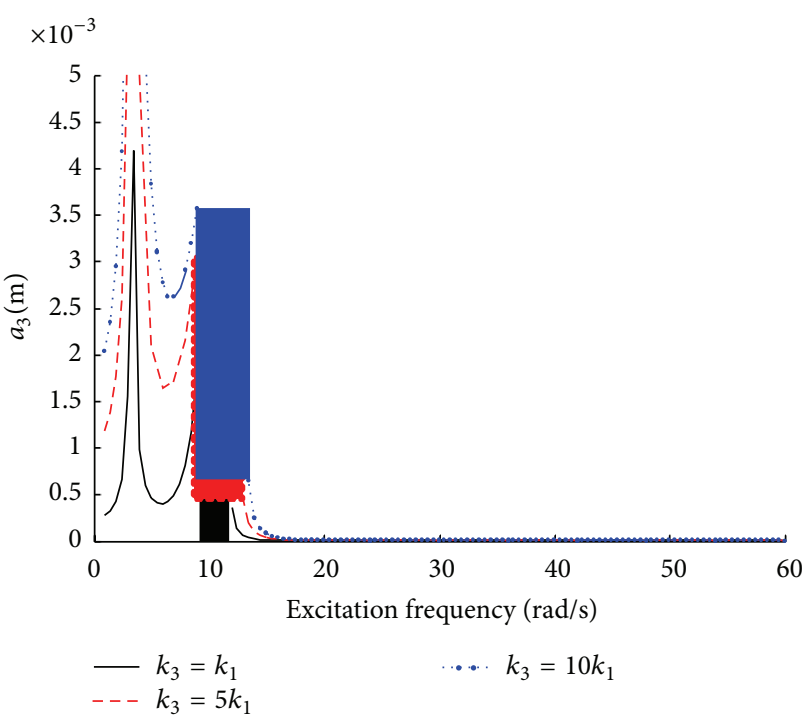

(b)

FIGURE 6: Influence of the nonlinear stiffness on the vibration properties of the oscillator; the shadow range is the unstable range: (a) the influence on $a_{1}$ and (b) the influence on $a_{3}$.

and the nonlinear stiffness just changes the amplitudes of oscillator below $2 \omega_{0}$. Hence, it could neglect the effects of the plate's vibration on the oscillator in most frequency bands.

\section{Conclusion}

This paper proposes an approximate analytical solution of the nonlinear vibroacoustic coupling system based on the IHB method and the modal expression method; the present method is straightforward, efficient, and reliable, and it can be used to obtain the nonlinear phenomena for the nonlinear vibroacoustic coupling system. The research on the Duffing oscillator-plate-medium system shows that when the excitation frequency is close to the natural frequency of the oscillator, the vibroacoustic characteristics of the plate are determined by the third-harmonic generation. In order to control the vibration and acoustic radiation of the system, the excitation frequency should be five times the natural frequency of the oscillator. The unstable range of the oscillator becomes wide due to the vibroacoustic coupling. The results could be used for the vibroacoustic design of the nonlinear oscillator. 


\section{Appendices}

\section{A. Acoustic Radiation Resistances}

The self-radiation resistances are

$$
\begin{aligned}
Z_{1 m n}=\xi_{1 m n}+i \chi_{1 m n} & \\
=\frac{\omega \rho}{2 \pi} \int_{0}^{a} \int_{0}^{b} \int_{0}^{a} \int_{0}^{b}( & \sin k_{m} x \sin k_{n} y \sin k_{m} x^{\prime} \sin k_{n} y^{\prime} \\
& \left.\quad \times \frac{e^{-i k\left|r-r^{\prime}\right|}}{\left|r-r^{\prime}\right|}\right) d x^{\prime} d y^{\prime} d x d y,
\end{aligned}
$$

$$
\begin{aligned}
Z_{3 m n}= & \xi_{3 m n}+i \chi_{3 m n} \\
=\frac{\omega \rho}{2 \pi} \int_{0}^{a} \int_{0}^{b} \int_{0}^{a} \int_{0}^{b}( & \sin k_{m} x \sin k_{n} y \sin k_{m} x^{\prime} \sin k_{n} y^{\prime} \\
& \left.\times \frac{e^{-i 3 k\left|r-r^{\prime}\right|}}{\left|r-r^{\prime}\right|}\right) d x^{\prime} d y^{\prime} d x d y .
\end{aligned}
$$

The calculation of the acoustic impedance is detailed in [4].

\section{B. Matrices in (19)}

The matrix $\mathbf{K}$ is

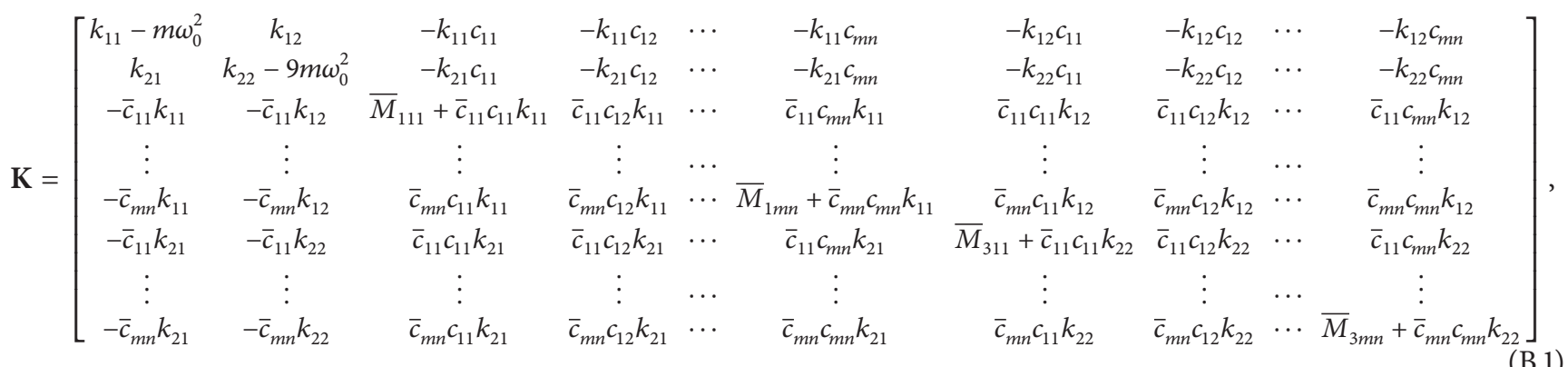

where $k_{11}=k_{1}+k_{3}\left((9 / 4) \bar{a}_{1}^{2}+(3 / 2) \bar{a}_{3}^{2}+(3 / 2) \bar{a}_{1} \bar{a}_{3}\right), k_{12}=$ $k_{3}\left((3 / 4) \bar{a}_{1}^{2}+3 \bar{a}_{1} \bar{a}_{3}\right), k_{21}=k_{12}, k_{22}=k_{1}+k_{3}\left((3 / 2) \bar{a}_{1}^{2}+(9 / 4) \bar{a}_{3}^{2}\right)$, $\bar{Z}_{1 m n}=b_{m n}-4 \omega_{0} Z_{1 m n} / a b D-\bar{m} \omega_{0}^{2}$, and $\bar{M}_{3 m n}=b_{m n}-$ $4 \omega_{0} Z_{3 m n} / a b D-9 \bar{m} \omega_{0}^{2}$.

The vector $\mathbf{R}$ is

$$
\begin{aligned}
& \mathbf{R}=\left[R_{1}+2 m \omega_{0} a_{1} \Delta \omega, R_{3}+18 m \omega_{0} a_{3} \Delta \omega,\right. \\
& R_{111}+\left(2 \bar{m} \omega_{0}-\frac{4 Z_{111}}{a b D}\right) a_{111} \Delta \omega, \\
& R_{112}+\left(2 \bar{m} \omega_{0}-\frac{4 Z_{112}}{a b D}\right) a_{112} \Delta \omega, \ldots, \\
& R_{1 m n}+\left(2 \bar{m} \omega_{0}-\frac{4 Z_{1 m n}}{a b D}\right) a_{1 m n} \Delta \omega, \\
& R_{311}+9\left(2 \bar{m} \omega_{0}-\frac{4 Z_{311}}{a b D}\right) a_{311} \Delta \omega, \\
& R_{312}+9\left(2 \bar{m} \omega_{0}-\frac{4 Z_{312}}{a b D}\right) a_{312} \Delta \omega, \ldots, \\
& \left.R_{3 m n}+9\left(2 \bar{m} \omega_{0}-\frac{4 Z_{3 m n}}{a b D}\right) a_{3 m n} \Delta \omega\right]^{T} .
\end{aligned}
$$

\section{Conflict of Interests}

The authors declare that there is no conflict of interests regarding the publication of this paper.

\section{Acknowledgment}

This work was supported by National Natural Science Foundation of China (Grant no. 11372350).

\section{References}

[1] L. Rayleigh, The Theory of Sound, Macmillan, New York, NY, USA, 2nd edition, 1896, (reprinted by Dover, New York, 1945).

[2] G. Maidanik, "Response of ribbed panels to reverberant acoustic fields," Journal of the Acoustical Society of America, vol. 34, pp. 809-826, 1962.

[3] E. G. Williams, "A series expansion of the acoustic power radiation from planar sources," Journal of the Acoustical Society of America, vol. 73, no. 5, pp. 1520-1524, 1983.

[4] W. L. Li and H. J. Gibeling, "Determination of the mutual radiation resistances of a rectangular plate and their impact on the radiated sound power," Journal of Sound and Vibration, vol. 229, no. 5, pp. 1213-1233, 2000.

[5] W. L. Li, "An analytical solution for the self- and mutual radiation resistances of a rectangular plate," Journal of Sound and Vibration, vol. 245, no. 1, pp. 1-16, 2001.

[6] I. V. Andrianov, V. V. Danishevs'Kyy, and J. Awrejcewicz, "An artificial small perturbation parameter and nonlinear plate 
vibrations," Journal of Sound and Vibration, vol. 283, no. 3-5, pp. 561-571, 2005.

[7] P. Ribeiro, "Nonlinear vibrations of simply-supported plates by the p-version finite element method," Finite Elements in Analysis and Design, vol. 41, no. 9-10, pp. 911-924, 2005.

[8] X. X. Wang, J. Qian, and M. K. Huang, "A boundary integral equation formulation for large amplitude nonlinear vibration of thin elastic plates," Computer Methods in Applied Mechanics and Engineering, vol. 86, no. 1, pp. 73-86, 1991.

[9] D. G. Crighton and G. Maidanik, "Acoustic and vibration fields generated by ribs on a fluid-loaded panel, I: plane-wave problems for a single rib," Journal of Sound and Vibration, vol. 75, no. 3, pp. 437-452, 1981.

[10] D. G. Crighton, “The 1988 Rayleigh medal lecture: fluid loadingThe interaction between sound and vibration," Journal of Sound and Vibration, vol. 133, no. 1, pp. 1-27, 1989.

[11] C. B. Burroughs and J. E. Hallander, "Acoustic radiation from fluid-loaded, ribbed cylindrical shells excited by different types of concentrated mechanical drives," Journal of the Acoustical Society of America, vol. 91, no. 5, pp. 2721-2739, 1992.

[12] A. Mejdi and N. Atalla, "Vibroacoustic analysis of laminated composite panels stiffened by complex laminated composite stiffeners," International Journal of Mechanical Sciences, vol. 58, no. 1, pp. 13-26, 2012.

[13] M. Chen, B. Wang, and W. Cao, "Research on vibration and acoustic radiation of the submerged complicated shell structure with multiple excitations," Acta Acustica, vol. 34, no. 6, pp. 498505, 2009.

[14] S. Wiggins, Introduction to Applied Nonlinear Dynamical Systems and Chaos, Springer, New York, NY, USA, 1990.

[15] S. L. Lau, Incremental harmonic balance method for nonlinear structural vibrations [Ph.D. thesis], University of Hong Kong, Hong Kong, 1982.

[16] S. L. Lau, Y. K. Cheung, and S. Y. Wu, "Nonlinear vibration of thin elastic plates, Part I: generalized incremental Hamilton's principle and element formulation, Part II: internal resonance," Journal of Applied Mechanics, Transactions ASME, vol. 51, no. 4, pp. 837-851, 1984.

[17] S. L. Lau and S. W. Yuen, "The Hopf bifurcation and limit cycle by the incremental harmonic balance method," Computer Methods in Applied Mechanics and Engineering, vol. 91, no. 1-3, pp. 1109-1121, 1991.

[18] C. W. Wong, Y. Q. Ni, and S. L. Lau, "Steady-state oscillation of hysteretic differential model. I: response analysis," Journal of Engineering Mechanics, vol. 120, no. 11, pp. 2271-2298, 1994.

[19] K. Y. Sze, S. H. Chen, and J. L. Huang, "The incremental harmonic balance method for nonlinear vibration of axially moving beams," Journal of Sound and Vibration, vol. 281, no. 35, pp. 611-626, 2005.

[20] K. C. Hall, K. Ekici, J. P. Thomas et al., "Harmonic balance methods applied to computational fluid dynamics problems," International Journal of Computational Fluid Dynamics, vol. 27, no. 2, pp. 52-67, 2013.

[21] Marcel Dekker, Thin Plates and Shells: Theory, Analysis, and Application, Marcel Dekker, New York, NY, USA, 2001.

[22] S. H. Chen, Quantitative Analysis Method of Strongly Nonlinear Vibration System, Science Press, Beijing, China, 2007, in Chinese. 

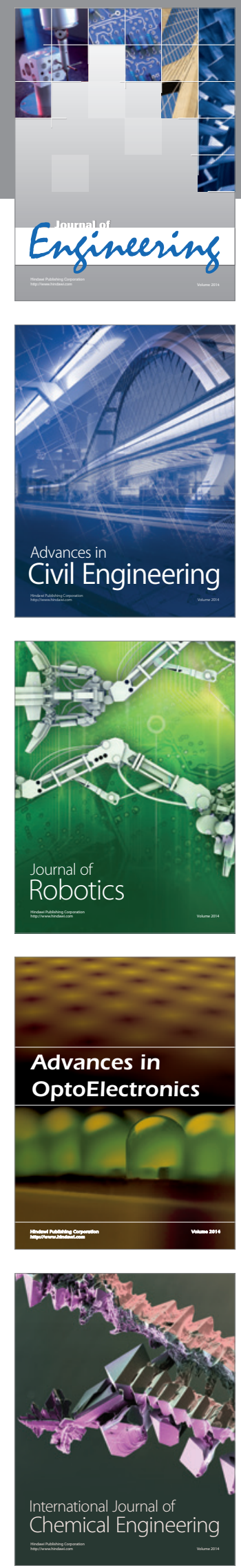

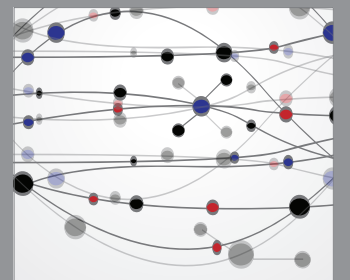

The Scientific World Journal


Submit your manuscripts at http://www.hindawi.com

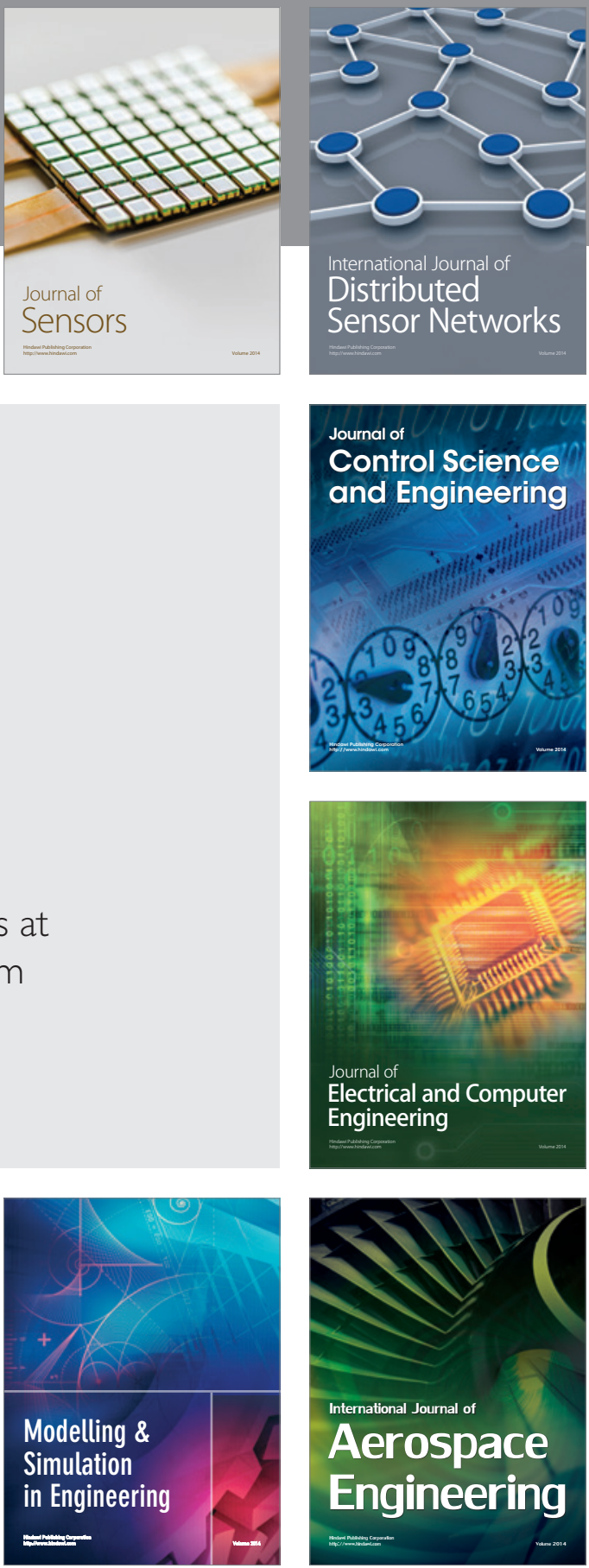

Journal of

Control Science

and Engineering
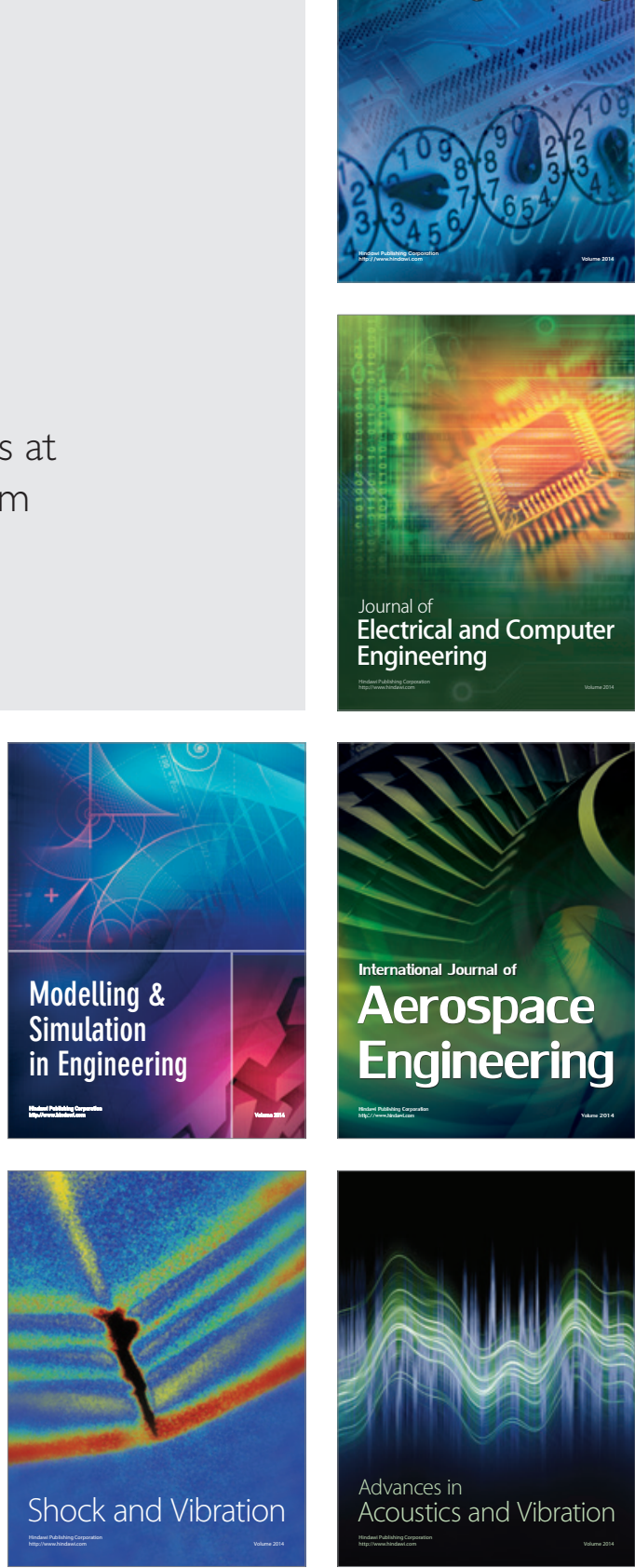